\title{
Entrelacs
}

Cinéma et audiovisuel

Nouvelles formes audiovisuelles documentaires

\section{Jouer le documentaire}

\section{Marine Bénézech et Michel Lavigne}

\section{OpenEdition}

\section{Journals}

Electronic version

URL: http://journals.openedition.org/entrelacs/1841

DOI: 10.4000/entrelacs. 1841

ISSN: 2261-5482

\section{Publisher}

Éditions Téraèdre

\section{Electronic reference}

Marine Bénézech et Michel Lavigne, « Jouer le documentaire », Entrelacs [Online], 12 | 2016, Online since 14 January 2016, connection on 20 April 2019. URL : http://journals.openedition.org/ entrelacs/1841; DOI : 10.4000/entrelacs.1841

This text was automatically generated on 20 April 2019.

Tous droits réservés 


\title{
Jouer le documentaire
}

\author{
Marine Bénézech et Michel Lavigne
}

1 Alors que se développent de nouvelles formes audiovisuelles interactives, les webdocumentaires permettent à l'utilisateur de gérer son parcours documentaire. Parmi ces objets, certains sont appelés « jeux documentaires » par leurs créateurs.

2 Cette irruption ludique dans le documentaire est à rattacher à un mouvement plus général d'extension du jeu dans nos sociétés numériques: il en est ainsi du concept de gamification consistant à injecter des procédés ludiques afin de rendre des applications du quotidien plus attractives (Jane McGonigal, 2011). Nous pouvons également identifier de nouveaux objets tels que les "serious games» (Alvarez et Djaouti, 2012) ou les « newsgames » qui, par le biais du jeu vidéo, tendent à proposer des « finalités sérieuses » pour les premiers ou à présenter des thématiques d'actualités, pour les seconds.

3 Alors que la question du jeu envahit des sphères de plus en plus larges de nos sociétés et s'applique à des domaines de plus en plus éloignés de ses attaches traditionnelles (monde de l'enfance, domaine de l'amusement), sa définition devient plus difficile à établir. Les éclairages apportés par les théoriciens classiques s'avèrent aussi insuffisants (Huizinga, 1988, Caillois, 1992). Ainsi, afin d'améliorer notre connaissance des jeux documentaires, nous éviterons de nous focaliser sur une définition préconçue. Nous nous attacherons à répondre aux questions suivantes : la mobilisation du jeu peut-elle véritablement rendre le documentaire jouable ? Le jeu peut-il enrichir l'expérience documentaire?

Nous partirons de l'étude sémio-pragmatique de jeux documentaires. Dans un premier temps, nous chercherons à montrer les éléments qui peuvent justifier leur qualification de jeu: des "marqueurs ludiques» qui, sans être des identifiants indiscutables de jouabilité, en sont tout au moins des indices. Dans un second temps, afin d'évaluer la jouabilité du documentaire par ses utilisateurs, nous confronterons un de ces produits à l'évaluation d'un public. Puis, nous élargirons ce point de vue en nous intéressant à l'utilisation de serious games basés sur de la vidéo afin d'examiner comment se configure «l'attitude ludique » (Henriot, 1989) face au jeu documentaire. 


\section{Analyse sémio-pragmatique de jeux documentaires}

5 Pour notre corpus, nous avons choisi cinq créations françaises de moins de cinq ans, définies comme des jeux documentaires par leurs créateurs. Elles explorent des modalités interactives, ludiques et documentaires différentes et paraissent ainsi représentatives de la diversité des jeux documentaires : Punk me de Jacques Denis, Narrative, Le Mouv, Radio France Nouveaux Médias, 2014 ; Jeu d'influences de Julien Goetz, France Télévisions et France Télévisions Nouvelles Ecritures, 2014 ; Réfugiés, un projet d'Arte GEIE, 2014 ; Fort Mc Money de David Dufresne, TOXA, ONF, Arte, 2013; Le défi des bâtisseurs, une coproduction SEPPIA - INDI FILM - ZDF - ARTE GEIE, 2012.

Pour analyser ces jeux documentaires, nous nous appuierons sur l'approche sémiopragmatique de Roger Odin (2011). Celui-ci envisage l'analyse d'un objet audiovisuel non seulement par l'examen de ses composants structuraux ou en fonction des intentions de l'auteur, mais aussi au regard du contexte de réception du spectateur. Cela nous conduira à cerner l'espace de communication producteur de sens au sein des jeux documentaires s'adressant moins à un spectateur qu'à un interacteur ou à un joueur. Au-delà de la présentation des sujets de ces objets et de leur analyse, nous nous attacherons à déterminer comment peut se forger la production de sens justifiant ce mode ludique, en inventoriant les marqueurs ludiques présents dans ces produits.

\section{Présentation des jeux et inventaire des marqueurs ludiques}

7 Conçu comme un parcours, Punk me propose de nous faire découvrir la culture et l'esprit punk. Punk me s'ouvre ainsi : « Y a-t-il un punk qui vit en toi ? Teste-toi pour le savoir... A toi de jouer ». L'internaute, immédiatement mis en position de joueur, n'est pas interrogé sur ses connaissances mais sur ses goûts. Les phases de tests sont accompagnées de dessins humoristiques. Cette légèreté de ton et la part de hasard de ce test manifestent une intention ludique, incitant l'internaute à naviguer spontanément. Il faut également relever le principe de ce test : toutes les étapes doivent avoir été accomplies, sans quoi il est impossible d'accéder au résultat. Sans être basé sur des niveaux à franchir, Punk me encourage l'internaute à participer à toutes les phases du test, l'invitant ainsi à suivre le documentaire jusqu'au bout. 


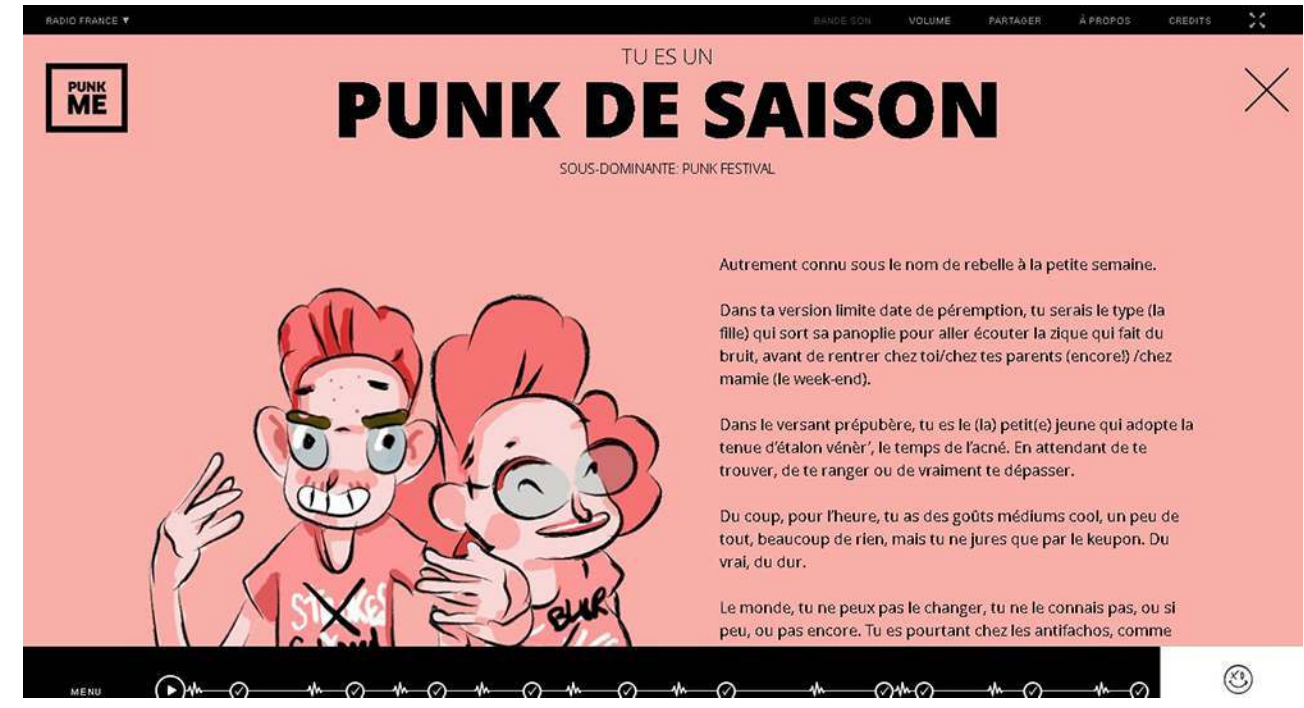

Figure 1 : Punk me. A la fin du test, l'internaute découvre son profil punk : un profil au second degré qui peut varier, selon les parties. L'internaute est invité à rejouer.

Dans Jeu d'influences, le personnage principal est fictif: Louis, directeur de la société Habinat, fait face à un scandale managérial et social. L'internaute est invité à se mettre dans la peau de ce directeur en péril et à faire les choix qu'il juge opportuns en matière de communication. L'apostrophe "Jusqu'où suivrez-vous votre spin-doctor?» que l'on découvre en introduction $\mathrm{du}$ jeu constitue tout autant son leitmotiv que sa règle. L'internaute est mis au défi. Jeu d'influences se divise en six chapitres, chacun constituant une étape de la crise. Pour passer d'un chapitre à l'autre, l'internaute est amené à faire des choix qui influenceront le cours des autres chapitres.

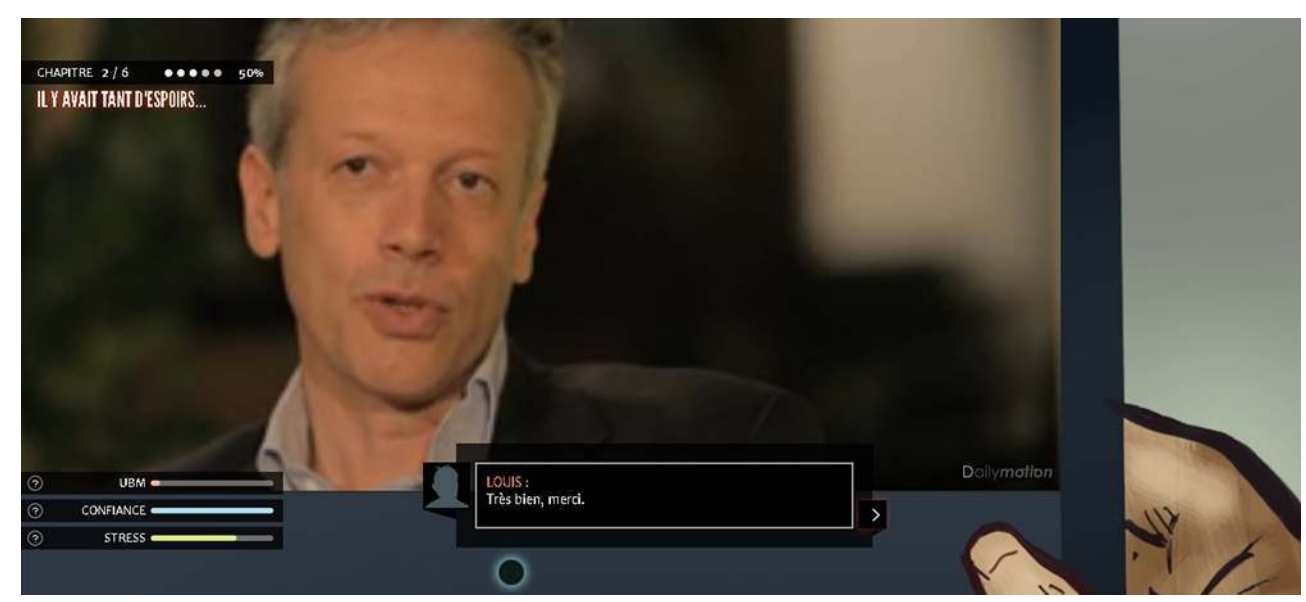

Figure 2 : Jeu d'influences. Pour faire ses choix, l'internaute peut consulter les documents fournis par le spin-doctor, comme des archives. A gauche, les jauges évaluent ses compétences.

9 On retrouve ici des caractéristiques du jeu vidéo linéaire: pour passer au chapitre suivant, il est nécessaire d'avoir franchi les étapes précédentes. Les choix de Louis influent sur le récit documentaire mais également sur son profil de joueur. Son stress, son émotivité et les Unités de Bruit Médiatique sont évalués par des jauges : si l'une des trois atteint 0 , Louis est «Game Over ». Ces jauges sont autant de marqueurs ludiques. Que le joueur aille au bout du jeu ou en soit exclu, un bilan lui est proposé : il peut alors « recommencer la partie ». Jeu d'influences fonctionne tel un jeu de stratégie où l'analyse de l'internaute permet le déroulement du récit. 
Le défi des bâtisseurs met en scène l'internaute dès les premiers plans : le jeu documentaire est filmé en vue subjective. L'internaute comprend qu'il s'agit de sa propre vision : l'un des personnages lui annonce qu'il est l'architecte que l'équipe attendait. Cette entrée en matière revêt un caractère ludique, dans la mesure où le « défi des bâtisseurs » devient le propre défi de l'internaute (construire la deuxième tour de la cathédrale de Strasbourg). L'internaute expérimente comme bon lui semble mais il doit cependant collecter suffisamment d'informations historiques et techniques pour réaliser une tour qui tienne debout. Nous sommes là dans un jeu d'aventure où l'internaute doit faire preuve de créativité et de stratégie : s'il ne sélectionne pas les bons matériaux ou ne s'associe pas aux bonnes personnes, il ne finalisera pas la tour.

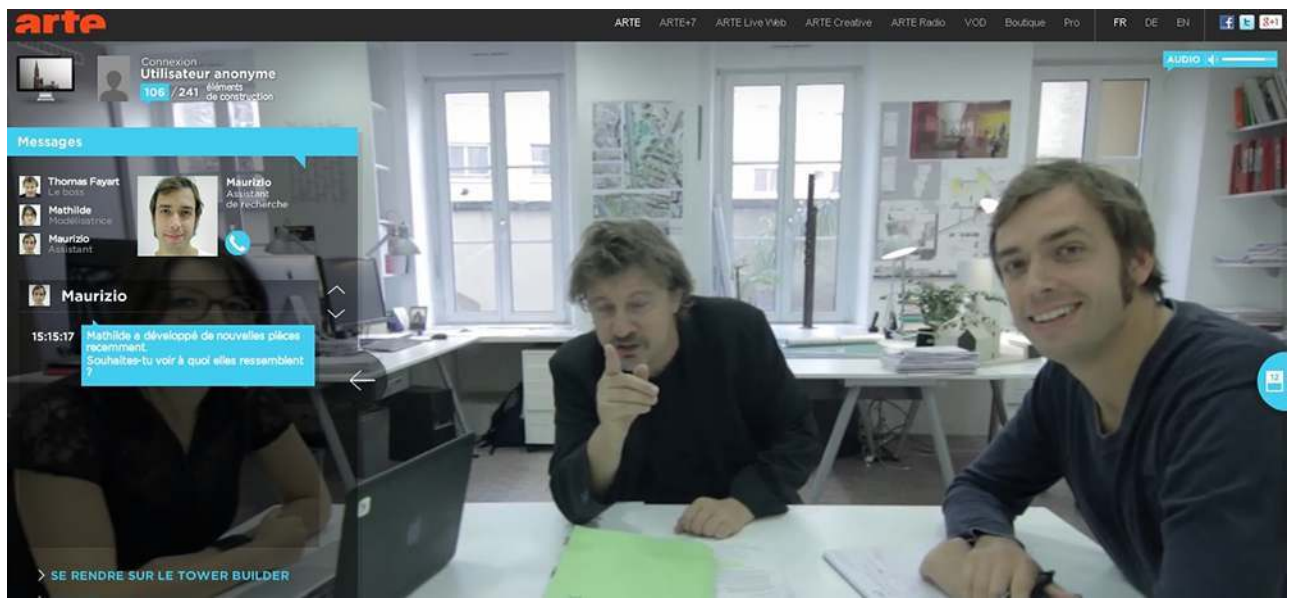

Figure 3 : Le défi des bâtisseurs. «C'est toi » : l'internaute, apostrophé par l'un des personnages, est immédiatement intégré au récit.

11 Réfugiés est un "reportage à jouer». La directrice des programmes d'Arte s'adresse directement à l'internaute et lui confie une mission: réaliser un documentaire. Ce que Réfugiés a de singulier, c'est qu'il n'y a pas de film à regarder. C'est à l'internaute de recueillir les informations (témoignages de réfugiés, ONG, etc.) et de "fabriquer un reportage ». Des contraintes sont imposées : temps limité, 5 supports documentaires à fournir, plusieurs lieux à explorer. Une horloge tourne en permanence. Ces marqueurs de jeux agissent comme des challenges à relever. Si le reporter sélectionne les mauvais témoins, il perd du temps et n'aura pas le nombre de supports requis pour monter son documentaire. Si celui-ci n'est pas conforme à la demande initiale, le reporter a perdu : le reportage ne sera pas diffusé par la chaîne. Les consignes de la directrice agissent comme des règles et le documentaire devient un jeu qu'il s'agit de gagner. 


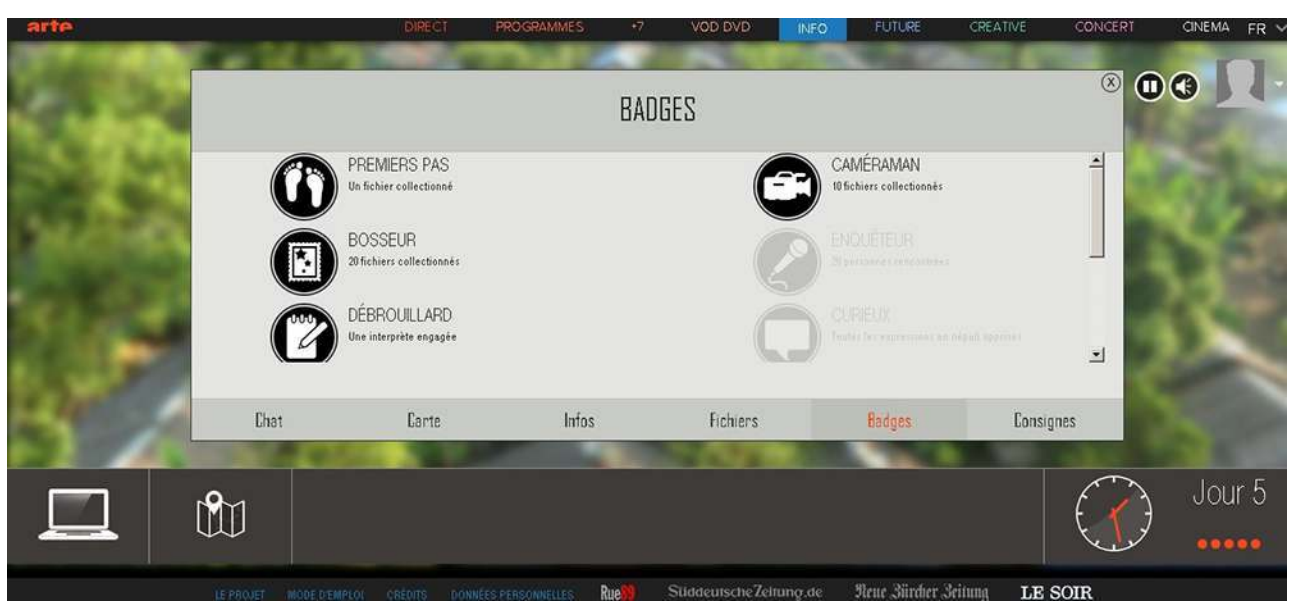

Figure 4 : Réfugiés. Des badges collectés, des compétences développées, une carte à explorer, du temps décompté : autant d'éléments inspirés de l'univers des jeux vidéo

Fort Mc Money débute par une voix-off : " vous entrez dans un jeu documentaire où tout est réel». L'objectif de Fort McMoney est de réunir des informations sur le fonctionnement d'une ville reposant sur l'économie du pétrole et de s'impliquer pour la faire évoluer. L'internaute observe et agit: il est son propre avatar et se déplace dans cette ville. Nous identifions clairement les caractéristiques des jeux vidéo. Le joueur dispose d'un tableau de bord dans lequel il retrouve tous les éléments qu'il a gagnés : cela participe à créer un enjeu de progression. Tous ces indices permettent d'accéder à de nouvelles ressources documentaires. Si l'internaute n'a ni exploré les bons lieux ni récolté les bonnes informations, il peut rester " coincé » : nous repérons là les codes du jeu d'aventure. L'internaute incarne la Lara Croft des forêts canadiennes. Ces passages d'un univers à un autre sont incarnés par les passages d'un écran à l'autre. L'internaute peut, par exemple, se saisir d'une télévision posée dans le décor : il accède à un autre univers, celui d'une archive documentaire. Dans Fort Mc Money, l'internaute est avant tout un explorateur dans un « monde ouvert » : il n'y a pas de fin attendue ou prévue.

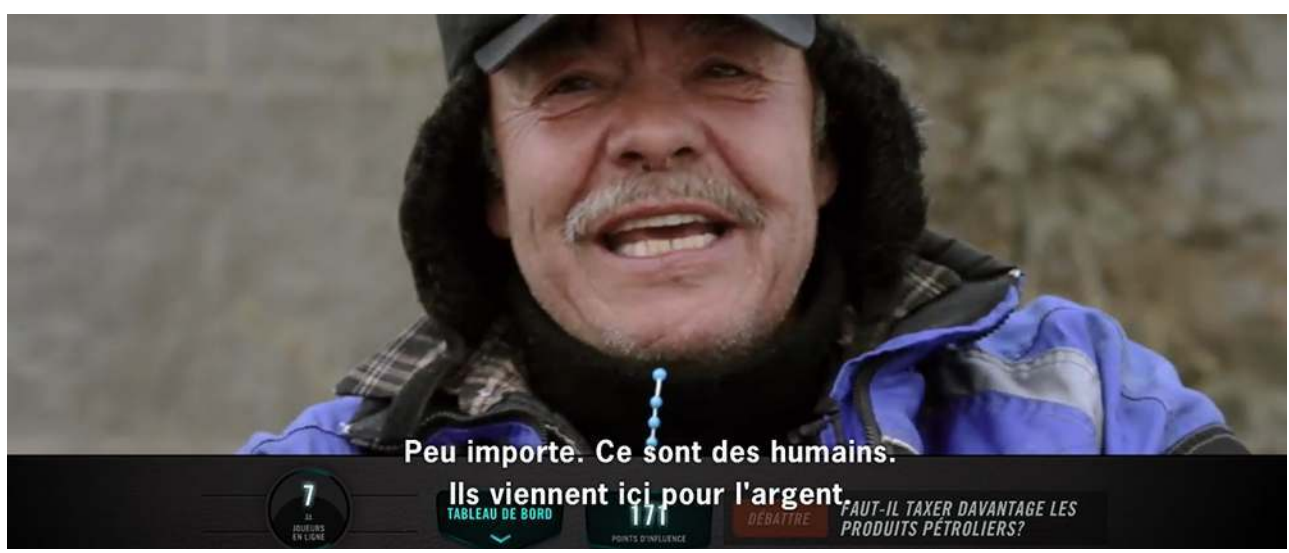

Figure 5 : Fort McMoney. L'internaute est invité à participer à un forum, visualise le nombre de points collectés et le nombre de joueurs connectés en même temps que lui. 


\section{Synthèse : les modalités ludiques des jeux documentaires: Mises en jeu(x) de l'internaute}

13 Dans ces jeux documentaires, l'internaute prend une part active dans le déroulement du récit. Au-delà d'un simple Point And Click, il est invité à s'impliquer personnellement. Cela se transcrit par une apostrophe, généralement dès la plateforme de lancement: «Teste-toi » (Punk me), "C'est toi que l'équipe attendait» (Le défi des bâtisseurs). L'emploi des pronoms personnels crée une proximité et encourage l'internaute à passer « de l'autre côté » de l'écran.

14 Ces interpellations facilitent ainsi la mise en action de l'internaute. Celle-ci revêt différentes formes. La première confine l'internaute dans son rôle; c'est en son nom qu'il intervient dans le récit. Dans Punk me, ses goûts sont "mis en jeu». Ses choix ne perturbent cependant pas la trame du documentaire : les tests s'effectuent parallèlement au récit. L'internaute peut donc suivre à la fois le documentaire et s'amuser à répondre aux questions, celles-ci composant des temps de pause. Ses actions aboutissent d'ailleurs à un bilan qui révèle (au second degré) sa personnalité.

15 L'autre forme identifiée propose à l'internaute de s'impliquer sous couvert d'une autre identité : il devient un acteur du récit, un personnage. Nous sommes ici dans un jeu de déguisement qui relève de la catégorie de la mimicry, si l'on se réfère à la classification de Roger Caillois (1992). Parce que l'internaute devient un personnage de l'histoire racontée, ses actions impactent celle-ci. Dans Jeu d'influence, un bilan est proposé : ce sont les choix de l'internaute qui sont évalués mais celui-ci agit en se mettant dans la peau du personnage qu'il incarne. Nous voyons bien que si l'internaute entre dans le jeu en son nom, il y progresse sous couvert d'un avatar. Le bilan, s'il dévoile bien des traits de personnalité de l'internaute, est le résultat de l'action du personnage principal.

16 Le procédé est poussé encore plus loin dans Fort McMoney. L'internaute demeure tout au long du jeu son propre avatar: ce dernier "prend le pouvoir». La mise en jeu personnalisante de l'internaute se révèle alors être un artefact, une simple incitation à cliquer et à entrer dans l'esprit du jeu.

17 Nous voyons que ce qui caractérise les jeux documentaires est bien l'implication personnalisée de l'internaute et que, au fur et à mesure des récits, celui-ci peut soit s'impliquer en son nom propre, soit sous couvert d'un avatar, ou même les deux en même temps.

\section{Structure linéaire ou récit éclaté}

18 Nous décelons deux formes narratives propres aux jeux documentaires. La première est linéaire. Dans Punk Me, tout comme dans Jeu d'influences, le documentaire est structuré avec un début, une progression et une fin. Les interventions de l'internaute n'influent que peu ou pas sur le déroulement de l'histoire. Cette forme narrative linéaire intègre des éléments ludiques reconnaissables avec des jauges évaluatives inspirées de l'univers des jeux vidéo. Ces jauges participent à créer un challenge et une tension. Ce sont ces éléments qui participent à faire de ces webdocumentaires des jeux documentaires. 
19 Ces jauges sont également présentes dans la deuxième forme narrative que nous avons identifiée : le « récit éclaté » (Réfugiés, Le défi des bâtisseurs, Fort Mc Money). L'internaute ne suit plus un récit linaire mais intègre un univers au sein duquel il choisit les portes qu'il pousse, les documents qu'il s'approprie. La démarche de l'internaute devient celle d'un explorateur. Tout comme le joueur de jeux vidéo, il franchit des étapes qui lui permettent d'avancer. Il est d'ailleurs intéressant de relever qu'à la fin de ces jeux documentaires, il n'est pas proposé de « revoir le documentaire », mais de « rejouer » ou de « recommencer la partie». Ces choix de vocabulaire impliquent qu'au contraire d'un deuxième visionnage, en rejouant la partie, l'internaute, en faisant des choix différents, découvrira d'autres documents. Les jauges et les tableaux de bord participent à analyser les choix et permettent, de ce fait, de rejouer différemment.

Structure linéaire et récit éclaté opposent ainsi deux modalités de récit : avec la structure linéaire, l'internaute actualise une histoire déjà écrite, même si les modalités de cette actualisation peuvent varier. Avec le récit éclaté, il devient le créateur d'une histoire particulière au sein d'une multiplicité de possibles.

\section{Des objectifs ludiques pluriels}

21 Nous relevons ainsi que les jeux documentaires peuvent revêtir des formes diverses, en fonction de la forme d'implication de l'internaute et de la structure narrative développée. Les jeux documentaires peuvent d'ailleurs également être différenciés selon les objectifs ludiques qu'ils proposent d'atteindre. La forme la plus courante propose au joueur de suivre un cheminement et de mener à bien une mission clairement définie. Nous remarquons ce procédé dans Le défi des bâtisseurs: l'internaute dispose d'une grande liberté d'action et peut mener la construction de la tour tel qu'il l'entend mais, quoi qu'il arrive, il doit la bâtir, sans quoi il perd. Dans Réfugiés, l'internaute sélectionne les sources qu'il souhaite mais doit coûte que coûte réaliser un documentaire pour réussir sa mission.

22 L'autre forme pourrait être identifiée comme une forme bac à sable : l'internaute évolue dans le jeu documentaire, au gré de ses envies. Il n'y a pas de but à atteindre ou de victoire à gagner. Il créé son propre univers au fur et à mesure des documents qu'il parcourt, des témoignages qu'il recueille, à l'instar de Fort McMoney. Ce type de jeu documentaire propose un monde sans fin qui se construit au fil du temps et dont l'évolution est imprévisible.

\section{Extension du domaine du jeu}

23 Nous pouvons cependant discerner que, quelle que soit la forme et l'objectif du jeu documentaire, une constante demeure: l'internaute est encouragé à poursuivre son exploration, au-delà de la plateforme initiale du documentaire et à partager ses résultats sur Facebook, Twitter, ou encore sur des forums thématiques. Toutefois, cette communication sur les réseaux sociaux ne présente pas le jeu documentaire sous l'angle documentaire, mais bel et bien sous celui du jeu: voici les résultats d'un joueur, quels seront les vôtres? Allez-vous participer? On remarque clairement l'intention de créer une " communauté de joueurs ». Cela se traduit par l'obligation de se créer un identifiant et un login pour se connecter: seuls ceux qui ont fait cette démarche auront accès au contenu, pourront participer aux forums de discussion et prendre part à des votes. Les 
internautes s'emparent du récit et le font évoluer par leurs décisions. Nous comprenons ainsi que cette forme d'extension transmédiatique du jeu documentaire permet à l'internaute de s'impliquer d'autant plus qu'il devient réellement acteur du jeu.

\section{Le jeu documentaire confronté à ses utilisateurs}

A la suite de l'évaluation sémiotique de jeux documentaires, il nous paraît nécessaire de les étudier en situation d'usage. Si le chercheur peut les analyser et les catégoriser, comment les publics les jugent-ils? Quelle peut en être l'appropriation? La fonction documentarisante est-elle améliorée ou entravée par la mise en scène ludique? Ces produits sont-ils ressentis plutôt comme des jeux, des documentaires ou des objets nouveaux?

\section{La démarche ethnométhodologique}

25 A la suite de travaux antérieurs portant sur l'évaluation de serious games (Lavigne, 2012, 2013, 2014), nous avons soumis le jeu documentaire Fort McMoney à l'évaluation d'un public étudiant, dans le cadre d'une enquête de type ethnométhodologique. Initiée par Harold Garfinkel, l'ethnométhodologie invite à décrire la réalité sociale au travers des pratiques des individus. Dans le domaine de l'analyse de logiciels, elle nous conduit à observer comment les utilisateurs se les approprient (ou les rejettent), les interprètent et le sens qu'ils donnent à leur expérience. Dans cette perspective, nous avons pratiqué l'analyse ethnométhodologique sur des corpus de serious games, en nous appuyant sur les expériences d'étudiants. Ces individus, représentatifs des jeunes générations nées avec les technologies numériques, sont porteurs d'une culture vidéoludique forgée par leur utilisation des jeux vidéo depuis leur enfance. Nous prenons pour hypothèse que ces jeunes partagent une culture commune en matière de jeu vidéo et que celle-ci s'appuie sur des pratiques, des procédures et un langage commun qu'il s'agit de questionner et de mettre à l'épreuve face à des serious games et dans le cas présent à un jeu documentaire.

Le public interrogé est constitué de groupes d'étudiants en 1ère année de DUT « Métiers du Multimédia et de l'Internet ». Par leur choix de formation, ils manifestent un intérêt pour les applications multimédia. Une grille d'analyse les conduit à décrire les divers aspects structuraux du produit: aspects techniques, esthétiques, ergonomie, structure narrative, finalité sérieuse, procédés ludiques. Nous leur demandons ensuite de procéder à une évaluation portant sur trois critères: qualité de l'univers (scénario, décors, personnages), qualité ludique (est-ce un jeu? est-il attractif?), efficacité sérieuse (message transmis). Cette évaluation se concrétise, pour chacun des critères, par l'attribution d'une note et d'appréciations qualitatives permettant de comprendre leurs motivations.

\section{La nature ludique de Fort McMoney}

Pour Fort McMoney, 57 fiches ont été documentées. Les notes attribuées aux trois critères sont de 15,40/20 pour l'univers, 14,79 pour l'aspect sérieux et seulement 9,57 pour la qualité ludique. Si les étudiants apprécient l'intrigue, la qualité de la mise en scène et l'apport d'informations «sérieuses », ils restent sceptiques sur la nature ludique de Fort 
McMoney. Par ailleurs, il est intéressant d'observer la variance de leurs réponses. Celle-ci est relativement faible pour la notation des univers $(5,54)$, nettement plus élevée pour l'efficacité sérieuse $(10,81)$ et encore plus pour la qualité ludique $(12,88)$. Les étudiants sont plutôt d'accords pour évaluer les aspects esthétiques et scénaristiques mais leurs critères divergent beaucoup pour évaluer l'apport documentaire et plus encore pour juger de l'aspect ludique.

Pour 52 de ces étudiants, nous disposons de données issues d'un questionnaire rempli lors d'une séance antérieure sur leurs pratiques des jeux vidéo, notamment le nombre d'heures hebdomadaires passées à jouer aux jeux vidéo. A partir de ces résultats, nous pouvons scinder cet effectif en 2 groupes: un groupe Grands Joueurs (GJ) dont les membres déclarent jouer au moins 10 heures par semaine (toutes pratiques de jeux vidéo confondues) et un groupe Petit Joueurs (PJ) qui déclarent jouer moins de 10 heures, voire pas du tout. Lors de l'évaluation de Fort McMoney, le groupe PJ est représenté par 28 étudiants. Le groupe GJ comprend 24 étudiants. Nous constatons une différence de la notation de la qualité ludique : 10,04 de moyenne pour les PJ, 9,25 pour les GJ. Ce résultat rejoint des constatations faites antérieurement lors de l'évaluation de serious games: plus un individu joue aux jeux vidéo, plus il a des attentes précises en la matière. A l'inverse, petits et non joueurs ont des critères moins arrêtés, sont moins exigeants et plus facilement enclins à accepter de «ranger » dans le monde du jeu toutes sortes de propositions.

Les données qualitatives recueillies permettent de préciser les approches et réactions des étudiants en nous focalisant sur les retours sur la question ludique. Comme nous l'avons mentionné plus haut, les étudiants sont très partagés dans leurs jugements sur ce critère. Aussi nous trouvons des avis positifs : c'est un «jeu attractif car on obtient différents reportages », il y a « du plaisir ludique car c'est un monde immersif ».

Les avis négatifs ou réservés sont néanmoins majoritaires: "aucun divertissement", "pas de plaisir ludique ", " on ne s'amuse pas ». Les motivations du jugement prennent des formes diverses: "ce n'est pas un jeu car il y a surtout des dialogues », "pas de challenge », il n'y a "pas de but ou d'objectif à atteindre pour gagner ou perdre ». Un certain nombre d'étudiants mettent en question la forme documentaire qu'ils estiment s'opposer au jeu : «la structure nous rend surtout spectateur ", " on reste assez passif », c'est « plus un documentaire qu'un jeu, il informe et ne divertit pas».

\section{Des jeux documentaires aux serious games basés sur la vidéo}

31 Depuis plusieurs années, nous remarquons qu'au fil de nos enquêtes ce type de critiques intervient fréquemment et particulièrement pour des serious games construits sur un matériau vidéo. Ces produits obtiennent généralement, en termes de qualité ludique, des notes inférieures à ceux construits à base d'univers tridimensionnels navigables ou en animation interactive. Nous pouvons rapprocher les critiques faites à Fort McMoney à celles adressées à des serious games basés sur de la vidéo tel que Premiers combats qui repose sur un univers de type série télévisée : ce n'est « pas ludique ou interactif, trop de vidéos, on est spectateur ", " on peut se demander si c'est un jeu, ça ressemble plus à un film », « l'histoire est prenante mais l'implication minime », « on subit l'histoire ». Pour Secret cam handicap «le joueur est plus spectateur que joueur ", "c'est comme si on 
regardait un film », «tout est plus ou moins prévu d'avance, les images sont des vidéos pré-enregistrées ». Pour Ce soir il conclut, c'est « plus une histoire interactive qu'un jeu ».

Par ces comparaisons, nous pensons que la vidéo incite fréquemment à une perception antinomique à celle du jeu, cette forme restant intrinsèquement attachée à la narration et à l'attitude spectatorielle. A l'inverse, les modalités ludiques relèvent de la mise en activité et de l'interactivité. De nombreux jeux vidéo contiennent des séquences en vidéo dénommées cinématiques. Ces jeux utilisent la vidéo sur un mode alternatif: les cinématiques sont le plus souvent considérées comme un temps de repos, de récompense, de reconstitution de l'énergie pour repasser à l'action. Cette opposition jeu / cinématique est particulièrement ancrée chez les grands joueurs. Il y a souvent une commande qui permet de passer la cinématique pour se replonger dans l'action. Un joueur reproche l'absence de cette commande dans Secret cam handicap: «on ne peut pas passer les cinématiques et elles sont longues ».

33 Avec les jeux documentaires, la manipulation des fragments vidéo constitue le plus souvent le principe du jeu lui-même. Nous ne sommes plus dans un mode alternatif jeu / vidéo, mais dans un mode intégratif. Cette perspective se heurte à divers obstacles. Le premier se trouve dans la perception de la vidéo par le public qui la place généralement en opposition au jeu, de la même façon qu'il oppose la narration qui s'adresse à un spectateur à l'interaction qui convoque un acteur. Pour beaucoup de joueurs, la vidéo contrarie par nature la forme ludique.

Le deuxième obstacle est d'ordre technique. Les joueurs souhaitent la maîtrise du jeu, l'exercice des possibles (Henriot, 1989) et une réponse immédiate du système à leurs sollicitations. De ce point de vue, il faudrait que la vidéo intègre un mode ludique en cours de visualisation, afin de la manipuler en permanence et de pouvoir la modifier à la volée au gré de ses désirs. Cela nécessiterait le tournage d'un nombre considérable de séquences et micro-séquences de manière à ce que le film contienne une infinité d'alternatives, de la même façon que les programmeurs permettent une infinité de possibilités dans les situations en $3 \mathrm{~d}$ temps réel.

\section{Conclusion}

Les analyses sémio-pragmatiques réalisées sur des jeux documentaires nous ont permis de relever que ces objets sont empreints de marqueurs ludiques incitant l'internaute à s'approprier le documentaire en tant que forme jouable. Nous soulignons cependant que ces marqueurs sont de "premier niveau» : des jauges, de l'humour, des dessins, etc. Ces éléments ludiques participent à rendre le documentaire attractif; ils peuvent offrir des challenges à relever. Le jeu n'apparaît donc pas comme l'objectif principal des jeux documentaires mais comme un moyen supplémentaire de motiver l'internaute et d'enrichir le documentaire.

Nous avons vu que les modalités ludiques sont diverses selon la place accordée au joueur et la structure adoptée. Ces modalités peuvent conduire d'une simple attractivité supplémentaire à la mise en jeu d'une réelle identification : cela pourrait transformer le documentaire en une expérience à vivre en lieu et place d'un récit à assimiler. La question qui reste alors posée est celle de l'efficacité de ces mises en scène : le jeu participe-t-il réellement d'une meilleure approche du documentaire? Peut-il favoriser un plus grand intérêt des nouveaux publics accoutumés aux jeux vidéo? Les enquêtes 
ethnométhodologiques que nous avons menées sont peu convaincantes de ce point de vue puisqu'elles révèlent que moins de la moitié des personnes sondées ressentent l'aspect ludique du jeu documentaire évalué, alors que les trois quarts perçoivent l'intention documentaire. Cette constatation rejoint celle effectuée lors de l'examen d'autres serious games basés sur de la vidéo. Les publics actuels ont du mal à reconnaître la nature ludique de ce type de produits.

Cet état de fait doit cependant être nuancé. En effet, les publics sollicités ne portaient pas d'intérêt particulier pour les thèmes proposés dans les jeux documentaires et serious games testés. Qu'en serait-il avec des publics ayant déjà une approche de ces sujets? Seraient-ils plus sensibles à la forme ludique ou la percevraient-ils comme un obstacle ? Par ailleurs, nous avons vu que, selon les pratiques habituelles de jeu, sa perception peut être très différente. La question de la perception ludique apparaît comme très personnelle. L'impact de l'ajout d'une scénarisation ludique dans une thématique documentaire peut avoir des effets imprévisibles selon les individus.

Au-delà des considérations subjectives individuelles, nous pouvons aussi interroger la perception sociale du jeu. Ainsi que le développe Jacques Henriot (1989), le jeu a vu sa signification évoluer selon les époques. Si le jeu défini par Huizinga (1988) ou Caillois (1992) reste très attaché à la question du divertissement, celui défini par Henriot est étendu, intégrant des questions très « sérieuses» telles que le «jeu social» ou le «jeu politique ». L'arrivée du jeu vidéo conduit encore à une évolution de la perception ludique basée sur de nouveaux dispositifs techniques qui imposent leurs codifications et les étendent, comme nous l'avons vu, à d'autres formes audiovisuelles, tel le jeu documentaire. Nous sommes ainsi en présence d'une forme de ludicisation du documentaire, pour reprendre le concept de Sébastien Genvo (2013).

Si pour certains, la forme ludique du webdocumentaire peut aujourd'hui apparaître comme un leurre, elle pourrait, à l'avenir, constituer une nouvelle modalité d'accès à l'information, accessible notamment à des populations connectées aux réseaux sociaux et aux communautés de joueurs. Le jeu participerait alors à un élargissement des formes audiovisuelles documentaires ouvrant ainsi la porte à de nouvelles possibilités immersives.

\section{BIBLIOGRAPHY}

\section{WEBOGRAPHIE}

Ce soir il conclut : www.cesoirilconclut.com/

Fort Mc Money : www.fortmcmoney.com

Jeu d'influences : jeu-d-influences.france5.fr/

Le défi des bâtisseurs : http://cathedrale.arte.tv/webdoc/\#/fr/home/

Premiers combats : http://www.premierscombats.com/seriousgame

Punk me : punk.lemouv.fr/ 
Réfugiés : http://refugees.arte.tv/fr/missions

Secret cam handicap : www.seriousgamesecretcam.fr/

BIBLIOGRAPHIE

Alvarez Julian, Djaouti Damien (2012), « Introduction au serious game », Questions théoriques.

Bogost Ian (2012), Newsgames - Journalism at Play, MIT Press.

Caillois, Roger (1992), Les jeux et les hommes, Gallimard, (1ère édition 1958).

Garfinkel Harold (2007), Recherches en ethnométhodologie, PUF.

Genvo Sébastien (2013), Penser la formation et les évolutions du jeu sur support numérique, Mémoire pour l'habilitation à diriger des recherches en sciences de l'information et de la communication, Université de lorraine.

Henriot Jacques (1989), Sous couleur de jouer, Paris, José Corti.

Huizinga Johan (1988), Homo ludens, Gallimard, (1ère édition 1951).

Lavigne Michel,

(2014), « Disséminations vidéoludiques : sous le masque du jeu », Interfaces numériques, $\mathrm{n}^{\circ} 3 / 3$, Lavoisier, pp. 473-496.

(2013), « Pertinence et efficacité des serious games. Enquête de réception sur neuf serious games ", RIHM, vol. 14, ${ }^{\circ}$ 1, [en ligne] URL : http://europia.org/RIHM/V14N1/3-RIHM-14\%281\% 29-Lavigne.pdf

(2012), «Serious games : que devient le plaisir ludique ?», Colloque Ludovia, août 2012, [en ligne] URL : culture.numerique.free.fr/publications/ludo12/lavigne_ludovia_2012.pdf

McGonigal Jane (2011), Reality is Broken: Why Games Make Us Better and How They Can Change the World, Penguin Books.

Odin Roger, (2011), Les espaces de communication : introduction à la sémio-pragmatique, PUG, Communication en plus.

\section{ABSTRACTS}

Some of webdocumentaries are also called documentary games by their makers. This article aims at analyzing the particularities of this kind of webdocumentaries and at wondering whether such an association between the game and the documentary is relevant. To this end, we first apply documentary game collection to a semio-pragmatic analysis, directing our attention to the recreational triggers found out in these productions. This analysis allows us to characterize different kinds of documentary games according to the web surfer's commitment, the structure of the programme and its purpose. Secondly, within the framework of an ethnomothological investigation, we confront a documentary game to an audience. The results highlight the difficulties perceiving the recreational aspect, and lead to wonder about the complexity of the fun in computer games as well in its subjective dimensions as in its social ones.

Certains webdocumentaires sont également qualifiés de jeux documentaires par leurs créateurs. Cet article a pour objet d'analyser les particularités de ce genre et de s'interroger sur la pertinence de la rencontre entre le jeu et le documentaire. A cette fin, dans un premier temps, nous soumettons un corpus de jeux documentaires à une analyse sémio-pragmatique en portant notre attention sur les marqueurs ludiques présents dans ces productions. L'analyse de ces 
éléments nous permet de caractériser diverses formes de jeux documentaires en fonction de la mise en jeu de l'internaute, de la structure du programme et de sa finalité. Dans un second temps, nous confrontons un jeu documentaire à un public, dans le cadre d'une enquête ethnométhodologique. Les résultats font ressortir les difficultés de la perception ludique et conduisent à interroger la complexité des déterminants ludiques dans leur dimension tant subjective que sociale.

INDEX

Mots-clés: webdocumentaires, jeux, documentaires, marqueurs ludiques, analyse sémiopragamatique, enquête ethnométhodologique 